\title{
Parallel computing technologies
}

\author{
Victor Malyshkin
}

Published online: 22 April 2011

(C) Springer Science+Business Media, LLC 2011, corrected publication 2022

\section{Dear Colleagues,}

This special issue of the journal is devoted to Parallel Computing Technologies $(\mathrm{PaCT})$. Today the $\mathrm{PaCT}$ is the mainstream research area in parallel computing. Generally, Science of Parallel Computing Technologies studies laws of the combined use of the results obtained in many different research areas: pure and applied mathematics, computer science, hardware architectures and software technologies, subject domains, including results from any disciplines needed for proving a successful application of supercomputers in a certain application area. Contrary to research in a "narrow" area, where usually a pure mathematical theory can be developed, in the $\mathrm{PaCT}$ we deal with a wide range of practical problems where it is impossible to operate only with the notions of a high abstraction in the framework of a certain theory suitable for analysis. As a rule, we are forced to operate simultaneously with the notions from different levels of abstraction as well as from the different theories. This demands the development of special approaches to the software development. The basic desirable result here is a certain concrete method helping to understand how to cross a gap between pure ideas via mathematical model and a necessary practical technology.

With the use of modern supercomputers it is now possible to develop and to implement on supercomputers different realistic mathematical models of natural phenomena, complex technical devises and human intellectual activities. In the area of mod-

This editorial is in reference to a special issue that was published in Volume 57, Issue 2, August 2011. Due to an oversight in the production process it was missed to publish it in the issue at the time.

V. Malyshkin ( $\varangle)$

Supercomputer Software Department, Institute of Computational Mathematics and Mathematical Geophysics, Russian Academy of Sciences, Novosibirsk, Russia

e-mail: malysh@ssd.sscc.ru 
eling, supercomputers are now irreplaceable and universal technological tool both in research and in practice. The attractiveness of studying nuclear explosions with modeling rather than observing real nuclear explosions seems to be clear to everybody.

The supercomputer applications meet serious problems. On the one hand, it is possible to assemble a supercomputer of a required performance for solving a desirable problem. On the other hand, there is a problem with developing and exploiting a necessary parallel program. Design, coding, debugging and maintenance of parallel programs are sophisticated tasks. This is a far more complex problem than the development of sequential programs. Sometimes in a simple, at first glance, program that has been already correctly exploited for a long time, fatal errors are found. The fact is that among numerous applications of supercomputers there are many applications sensible to errors. For this reason, in the PaCT much effort is being spent into development of the methods of automatic or automatized construction of totally correct parallel programs.

The present issue is composed based of the selected papers presented at the 10th International Conference on Parallel Computing Technologies (PaCT-2009) which was held in Novosibirsk, on August 31-September 4, 2009. All the papers were carefully revised; the recent results were also included. The papers included into this issue treat the models of parallel computing, methods and algorithms of parallel software development, fine-grained parallelism, parallel programming tools and supercomputer applications.

I would like to thank the members of the PaCT-2009 Program Committee for their help in selecting the papers and the authors of the papers for their support in preparing the issue. 